\title{
Oral Health Related Knowledge, Attitude And Behavior Among Group of Mothers In Relation To Their Primary School Children's Oral Health, Jeddah, Saudi Arabia: A Cross-Sectional Study
}

Eman A. El Ashiry

King Abdulaziz University

Najlaa M. Alamoudi

King Abdulaziz University

Nada Bamashmous

King Abdulaziz University

Rana A. Alamoudi ( $\square$ rasalamoudi@kau.edu.sa )

King Abdulaziz University

Duha K. Qahtan

Saudi Airlines Medical Services

Osama M. Felemban

King Abdulaziz University

\section{Research Article}

Keywords: Oral health, mothers, children, Knowledge, Attitude, Behavior, primary schools, dental caries, oral hygiene index.

Posted Date: December 14th, 2021

DOI: https://doi.org/10.21203/rs.3.rs-1155126/v1

License: (c) (i) This work is licensed under a Creative Commons Attribution 4.0 International License. Read Full License 


\section{Abstract}

Aim: To assess the knowledge, attitudes, and behavior of a group of mothers with their children aged 6-12 years regarding their own and their children's oral health and to evaluate its impact on their children oral health status in Jeddah, Saudi Arabia.

Methods: A validated, and reliable Arabic questionnaire comprised four sections (demographic characteristics and socioeconomic status, Hiroshima University- Dental Behavioral Inventory; HU-DBl; for mother' attitudes and behavior pertaining their own oral health, mothers' knowledge, and mothers' attitudes regarding oral health of their children) was used for this study. The questionnaire was completed by the mothers. A clinical oral examination was performed for the children, attending primary schools in Jeddah city, to detect the caries experience and Simplified Oral Hygiene Index (OHI-S).

Results: A total of 1496 mother-child pairs completed the study. The mean values of dmft were $4.08 \pm$ 3.47; DMFT was $1.82 \pm 2.07$; total dmft and DMFT was $5.65 \pm 4.05$; and the OHI-S was $1.17 \pm 0.94$. According to the questionnaire results, mothers in private schools had a more favorable attitude and behavior toward their own oral health, as well as a more favorable knowledge and attitude toward their children's oral health. The multiple linear regression model revealed that the children's dmft/DMFT scores were significantly related to mother education, mother questionnaire scores, and the OHI-S.

Conclusion: Mother education, knowledge, and attitude all play a role in the development of dental caries. Overall, mothers need to improve their oral health attitudes and behaviors, as well as increase their level of oral health knowledge.

\section{Introduction}

Although caries is considered a preventable disease, however, its worldwide prevalence remains high. In Saudi Arabia, prevalence of dental caries remained high reaching $97 \%$ as recently reported by the Ministry of health (1). Factors associated with the increased prevalence of childhood dental caries were investigated comprehensively in the literature. Some of the most important studied factors were those related to children's parents/caregiver. Parents play an important role in maintaining children's good oral health and establishing better oral health related habits. Parents' socioeconomic status, habits, knowledge, and attitude towards their children's oral health were reported to have a major impact on children's oral health (2).

The association between dental caries and socioeconomic status is well stablished (3-5). Children of fathers with higher socioeconomic status and non-working mothers were found to have lower caries prevalence compared to those with lower socioeconomic status $(6,7)$. Previous studies reported higher prevalence of poor oral health in children of mothers with low educational level (8-10). Additionally, it was reported that mothers with lower educational levels had poorer knowledge regarding their children oral health leading to increased incidence of early childhood caries (11). 
Parents tend to be their children's role model especially when it comes to the oral hygiene practice (12). An epidemiological study conducted in Belarus reported high prevalence of dental caries. The lack of parental knowledge and inconsistency between the parent's dental knowledge and their actual practice were one of their major findings and the focus for their recommendations (13). A systematic review was conducted to assess the effect of the parents' dental health habits on their children's oral health. They concluded that parent's dental habit can have an impact on their children's oral health (14). In Saudi Arabia, Alhabdan conducted a study in Riyadh to evaluate the association between parent behavior and attitudes toward their children's oral health and to relate it with the oral hygiene statues for their children. They found higher dental caries prevalence among children aged 6-8 years resulting from inadequate oral health attitude and behaviors of their parents (15).

The association between parent and their children's oral health has been investigated since 1946 (16) with stronger association among mothers than fathers (17). Hence, mothers' knowledge, attitude and behavior toward oral health need to be optimum to maintain their own and their children's oral health. Therefore, the aim of the study is to evaluate the level of oral health-related knowledge, attitudes, and behavior among a group of mothers with children aged 6-12 years toward their own and their children (oral health), and to assess its influence on the oral health status of their children in terms of dental caries experience.

\section{Methods}

Subjects: The sample was a multistage stratified random one consisting of children attending primary schools. A group of mothers with their children aged 6 - 12 years participated in the study. The children are healthy ASA I and their native language is Arabic. They are joining primary schools either private or public in Jeddah city, Saudi Arabia.

According to Ministry of Education files, the number of registered children in the primary schools in the academic year 2017-2018 was 293,373 children (147,525 boys and 145,848 girls) in 737 schools in Jeddah districts. Based on the gender, schools were divided into boys' schools (344) and girls' schools (393) following the country's regulations. Sample size was calculated based on previous research (18). A two-sided 95\% confidence interval for a single proportion using the large sample normal approximation modified for a finite population of size 300,000 will stretch 0.025 from the observed percentage for an expected proportion of $35 \%$ when the sample size is 1392 . The schools were stratified on three levels:

- Four strata, by district (west, east, north, south).

- Then by type each strata was stratified into public or private producing 8 strata.

- Finally, by gender each of the 8 strata was stratified into boys or girls resulting in 16 strata.

Random number generator (19) was used to select one schools from each of the 16 strata resulted into 16 primary schools with approximately 7,181 children from different regions in Jeddah. To compensate for the drop out of participated mother-child pairs due to incomplete questionnaire response or the child 
resistance to oral examination, 1728 questionnaires were distributed. From each grade, one class was randomly chosen to join the study by the bowl method. In cases of a small number of children in any class (less than 18), another class was randomly selected, and in cases of a small number of children in the school (less than 108), the whole school children were included in the sample.

\section{Questionnaire}

The questionnaire had a cover letter describing the aim of the study and stressing confidentiality of the responses. An Arabic questionnaire comprised of four sections was used for this study. The questionnaire was completed by mothers. Section I, to record the demographic characteristics like child gender; and mothers' age (40 years or less, 41years or more), educational level (High school or less, Diploma/University or higher) and monthly family income; (low, medium, or high). The informed consent was attached to this section. Section II, Hiroshima University- Dental Behavioral Inventory (HU-DBI)questionnaire, to evaluate attitudes and behaviors of mothers pertaining their own oral health based on the sum of agree/disagree responses by giving one point to each favorable response of good oral health (20). This section contains 20 items asking about the protection of her teeth, regular visits to the dentist, ideal toothbrush, and toothpaste. To clarify item 5 (I use child-sized toothbrush), it was modified; into (I use recommended-sized toothbrush). High scores indicate good oral health attitudes and behaviors (2129). Section III of the questionnaire was to recognize mothers' knowledge about their children oral health (30). The present study used the last 2 sections (12 questions) concerning dental health and dental care for children. Mothers selected the correct response from multiple choices. One point was given for each correct answer. Section IV for identifying the mothers' attitudes towards their children's oral health, (31). It was composed of 13 agree/disagree questions. One point was given for each correct answer.

Arabic Translation of the questionnaire was carried out by two Saudi linguists. Forward and backtranslation was used. The first linguist, who is fluent in both the Arabic and English languages, translated the questionnaire from English to Arabic, forward translation. The other linguist translated back the Arabic version into English language, backward translation. Then, a dental public health professor compared the two English questionnaires (the original one and translated one) and made modifications until the final Arabic translated version was formulated which was considered the final Arabic questionnaire (32).

Test-retest Reliability of the Arabic questionnaire was done. The final Arabic version of the questionnaire was completed twice with two weeks' interval by a group of mothers (30 mothers), not included in the study. The results were compared using Pearson's correlation coefficient (Pearson's $r$ ) as a reliability test. Pearson's $r$ between the two questionnaires' responses was referred to as the coefficient of stability and were found to equal 0.90 which is considered excellent. Moreover, internal consistency was done to reflect the inter-correlation between items in the questionnaire and was quantified using the Cronbach's alpha to be $0.86(33,34)$. 
Content validity of the Arabic translated version of the questionnaire was performed to recognize if the questionnaire items were representative of the entire theoretical construct that the questionnaire was proposed to measure. A panel of experts in pediatric dentistry were asked to rate each item in the questionnaire concerning relevance, clarity, simplicity, and ambiguity on a four-point Likert scale. The Content Validity Index (CVI) was calculated according to the methodology of Lynn MR and Polit et al and was found to be $0.84(33,34)$.

Three school visits were performed. The first visit for distribution of the questionnaire for the selected children in the class to submit to their mothers with the help of the class teacher. Each questionnaire was assigned a serial number which was matched with an examination sheet for confidentiality purposes. The second visit was carried out after one week, for collection of the questionnaires and clinical oral examination for the children who had a permission from their mothers to assess dental caries experience using two indices (dmft) and (DMFT) (35), and oral hygiene status of the children by Simplified Oral Hygiene Index (OHI-S); Debris Index component (DI); (Greene and Vermillion, 1964). A brief report was sent to the mothers describing the oral health status of their children and instructions for improvement and/or maintaining the oral health condition. Two groups of examiners, one for girls' schools (two examiners) and one for boys' schools (two examiners) shared in this study. Inter and intra-rater reliability test was done for Calibration and training of the examiners. Kappa statistic was calculated and found excellent ( 0.93 for female and 0.95 for male examiners). The inter-rater reliability between the examiners was evaluated using the Intra-class Correlation (ICC) and was found to be 0.96 . On the third school visit, mother-child pairs were gathered for presenting an oral health education lecture and distribution of oral health instruction pamphlets to increase their awareness of the proper oral health knowledge, attitude and behavior regarding themselves and their children.

The research protocol was approved by The Research Ethics Committee at the Faculty of Dentistry, King Abdulaziz University with proposal number (008-16). In addition, approval was obtained from the local School Health and Education Directorate Authority; Ministry of Education; to provide the primary schools' lists in Jeddah city districts and to implement the research among the schools.

\section{Statistical Analysis}

Data was entered, coded, and analyzed using the Statistical Package for Social Science SPSS (IBM Statistics for Windows, Version 23.0 Armonk NY: IBM Corp). Univariate analysis of data was carried out and presented as frequencies, percentages or means and standard deviations. The responses of the three questionnaires from public schools were compared to private schools using chi-square test. The association between the demographic variables and questionnaire score, DMFT, and OHI-S was evaluated using independent t-test or ANOVA test which was followed by post hoc analysis. A multiple linear regression was modelled to assess the effects of all the significant independent variables on the DMFT (dependent variable). The significance level was set as $\mathrm{p}<0.05$.

\section{Results}


The research comprised a total of 1496 mother-child pair out of the 1728 distributed questionnaires (a response rate of $86.57 \%)$. A little over half were from private schools $(50.2 \%)$. About half $(53.8 \%)$ of the children were aged $6-9$ years and $46.2 \%$ were aged $10-12$ years. Male children accounted for $47.1 \%$ and female children for $52.9 \%$. Mothers' age has been distributed according to the following figures: $56.2 \%$ were aged 40 years or less and $43.8 \%$ were aged 41 years or older. Only $44.3 \%$ of mothers had high school education or less whilst $55.7 \%$ had diploma, university, or higher education. Less than quarter of mothers had low monthly income (22.4\%), less than half had a medium income (46.7\%), and less than one third had a high income (30.9). Table 1 shows the demographic characteristics of children and mothers.

With respect to the children oral health, the average dmft was $4.08 \pm 3.47$; DMFT was $1.82 \pm 2.07$; total dmft and DMFT was $5.65 \pm 4.05$; and the Debris index was $1.17 \pm 0.94$.

Table 1

Demographic features of the research sample children and their mothers

\begin{tabular}{|lll|}
\hline Variable & Category & Frequency (\%) \\
\hline School type & Public & $745(49.8)$ \\
\cline { 2 - 3 } Child age & Private & $751(50.2)$ \\
\hline Child gender & $\mathbf{6 - 9}$ years & $805(53.8)$ \\
\cline { 2 - 3 } & $\mathbf{1 0 - 1 2 \text { years }}$ & $691(46.2)$ \\
\hline Mother's age & Male & $704(47.1)$ \\
\cline { 2 - 3 } & $\mathbf{4 0}$ or less & $792(52.9)$ \\
\cline { 2 - 3 } Mother's education & $\mathbf{4 1}$ or more & $841(56.2)$ \\
\cline { 2 - 3 } & High school or less & $655(43.8)$ \\
\cline { 2 - 3 } Monthly Income & Diploma/University or higher & $833(55.7)$ \\
\cline { 2 - 3 } & Low & $335(22.4)$ \\
\cline { 2 - 3 } & Medium & $698(46.7)$ \\
\cline { 2 - 3 } & High & $463(30.9)$ \\
\cline { 2 - 3 } & &
\end{tabular}

The replies of the mothers to the HU-DBI questionnaire are shown in Table 2. When comparing moms of children in public vs private schools, there was a statistically significant difference in 16 out of the 20 questions. Regarding replies to questions $6,8,9$, and 16 , the differences were not statistically significant between mothers who are enrolled their children in public school's vs those in private schools. Mothers in public schools had much higher favorable replies to questions 3,4 , and 13 compared to mothers in 
private schools. Mothers at private schools tended to provide more favorable replies to questions $1,2,5$, $7,10,11,12,14,15,17,18,19$, and 20. 
Table 2

Comparison between public and private schools of mothers' answers to the HUDBI questionnaire

\begin{tabular}{|c|c|c|c|c|c|c|}
\hline \multicolumn{2}{|c|}{ Items } & $\begin{array}{l}\text { The correct } \\
\text { answer }\end{array}$ & $\begin{array}{l}\text { Total } \\
\mathrm{n}=1496\end{array}$ & $\begin{array}{l}\text { Public } \\
n=745\end{array}$ & $\begin{array}{l}\text { Private } \\
\mathrm{n}=751\end{array}$ & $\begin{array}{l}\mathrm{P}- \\
\text { value }\end{array}$ \\
\hline 1 & $\begin{array}{l}\text { I don't worry much about visiting the } \\
\text { dentist }\end{array}$ & Agree & $\begin{array}{l}631 \\
(42.2)\end{array}$ & $\begin{array}{l}246 \\
(33.0)\end{array}$ & $\begin{array}{l}385 \\
(51.3)\end{array}$ & $<0.001$ \\
\hline 2 & $\begin{array}{l}\text { My gums tend to bleed when I brush } \\
\text { my teeth }\end{array}$ & Disagree & $\begin{array}{l}1096 \\
(73.3)\end{array}$ & $\begin{array}{l}495 \\
(66.4)\end{array}$ & $\begin{array}{l}601 \\
(80.0)\end{array}$ & $<0.001$ \\
\hline 3 & I worry about color of my teeth & Agree & $\begin{array}{l}933 \\
(62.4)\end{array}$ & $\begin{array}{l}554 \\
(74.4)\end{array}$ & $\begin{array}{l}379 \\
(50.5)\end{array}$ & $<0.001$ \\
\hline 4 & $\begin{array}{l}\text { I have noticed some white sticky } \\
\text { deposits on my teeth }\end{array}$ & Agree & $\begin{array}{l}113 \\
(7.6)\end{array}$ & $\begin{array}{l}82 \\
(11.0)\end{array}$ & $\begin{array}{l}31 \\
(4.1)\end{array}$ & $<0.001$ \\
\hline 5 & I use a recommended -sized toothbrush & Agree & $\begin{array}{l}1435 \\
(95.9)\end{array}$ & $\begin{array}{l}690 \\
(92.6)\end{array}$ & $\begin{array}{l}745 \\
(99.2)\end{array}$ & $<0.001$ \\
\hline 6 & $\begin{array}{l}\text { I think that I cannot help having false } \\
\text { teeth when I am old }\end{array}$ & Disagree & $\begin{array}{l}80 \\
(5.3)\end{array}$ & $\begin{array}{l}48 \\
(6.4)\end{array}$ & $\begin{array}{l}32 \\
(4.3)\end{array}$ & 0.061 \\
\hline 7 & I am bothered by the color of my gums & Disagree & $\begin{array}{l}1275 \\
(85.2)\end{array}$ & $\begin{array}{l}602 \\
(80.8)\end{array}$ & $\begin{array}{l}673 \\
(89.6)\end{array}$ & $<0.001$ \\
\hline 8 & $\begin{array}{l}\text { I think my teeth are getting worse } \\
\text { despite my daily brushing }\end{array}$ & Disagree & $\begin{array}{l}867 \\
(58.0)\end{array}$ & $\begin{array}{l}441 \\
(59.2)\end{array}$ & $\begin{array}{l}426 \\
(56.7)\end{array}$ & 0.333 \\
\hline 9 & I brush each of my teeth carefully & Agree & $\begin{array}{l}1283 \\
(85.8)\end{array}$ & $\begin{array}{l}630 \\
(84.6)\end{array}$ & $\begin{array}{l}653 \\
(87.0)\end{array}$ & 0.186 \\
\hline 10 & $\begin{array}{l}\text { I have never been professionally taught } \\
\text { how to brush }\end{array}$ & Disagree & $\begin{array}{l}466 \\
(31.1)\end{array}$ & $\begin{array}{l}169 \\
(22.7)\end{array}$ & $\begin{array}{l}297 \\
(39.5)\end{array}$ & $<0.001$ \\
\hline 11 & $\begin{array}{l}\text { I think I can clean my teeth without } \\
\text { using toothpaste }\end{array}$ & Agree & $\begin{array}{l}348 \\
(23.3)\end{array}$ & $\begin{array}{l}125 \\
(16.8)\end{array}$ & $\begin{array}{l}223 \\
(29.7)\end{array}$ & $<0.001$ \\
\hline 12 & $\begin{array}{l}\text { l often check my teeth in a mirror after } \\
\text { brushing }\end{array}$ & Agree & $\begin{array}{l}1092 \\
(73.0)\end{array}$ & $\begin{array}{l}494 \\
(66.3)\end{array}$ & $\begin{array}{l}598 \\
(79.6)\end{array}$ & $<0.001$ \\
\hline 13 & I worry about having bad breath & Agree & $\begin{array}{l}250 \\
(16.7)\end{array}$ & $\begin{array}{l}166 \\
(22.3)\end{array}$ & $\begin{array}{l}84 \\
(11.2)\end{array}$ & $<0.001$ \\
\hline 14 & $\begin{array}{l}\text { It is impossible to prevent gum disease } \\
\text { without toothbrushing alone }\end{array}$ & Disagree & $\begin{array}{l}523 \\
(35.0)\end{array}$ & $\begin{array}{l}214 \\
(28.7)\end{array}$ & $\begin{array}{l}309 \\
(41.1)\end{array}$ & $<0.001$ \\
\hline 15 & $\begin{array}{l}\text { I put off going to the dentist until I have } \\
\text { a toothache }\end{array}$ & Disagree & $\begin{array}{l}211 \\
(14.1)\end{array}$ & $\begin{array}{l}63 \\
(8.5)\end{array}$ & $\begin{array}{l}148 \\
(19.7)\end{array}$ & $<0.001$ \\
\hline 16 & $\begin{array}{l}\text { I have used a dye to see how clean my } \\
\text { teeth are }\end{array}$ & Agree & $\begin{array}{l}40 \\
(2.7)\end{array}$ & $\begin{array}{l}22 \\
(3.0)\end{array}$ & $\begin{array}{l}18 \\
(2.4)\end{array}$ & 0.505 \\
\hline
\end{tabular}




\begin{tabular}{|c|c|c|c|c|c|c|}
\hline \multicolumn{2}{|c|}{ Items } & $\begin{array}{l}\text { The correct } \\
\text { answer }\end{array}$ & $\begin{array}{l}\text { Total } \\
\mathrm{n}=1496\end{array}$ & $\begin{array}{l}\text { Public } \\
\mathrm{n}=745\end{array}$ & $\begin{array}{l}\text { Private } \\
\mathrm{n}=751\end{array}$ & $\begin{array}{l}\mathrm{P}- \\
\text { value }\end{array}$ \\
\hline 17 & $\begin{array}{l}\text { I use a toothbrush which has hard } \\
\text { bristles }\end{array}$ & Disagree & $\begin{array}{l}1243 \\
(83.1)\end{array}$ & $\begin{array}{l}573 \\
(76.9)\end{array}$ & $\begin{array}{l}670 \\
(89.2)\end{array}$ & $<0.001$ \\
\hline 18 & $\begin{array}{l}\text { I don't feel l've brushed well unless I } \\
\text { brush with strong strokes }\end{array}$ & Disagree & $\begin{array}{l}833 \\
(55.7)\end{array}$ & $\begin{array}{l}370 \\
(49.7)\end{array}$ & $\begin{array}{l}463 \\
(61.7)\end{array}$ & $<0.001$ \\
\hline 19 & $\begin{array}{l}\text { I feel I sometimes take too much time } \\
\text { to brush my teeth }\end{array}$ & Agree & $\begin{array}{l}903 \\
(60.4)\end{array}$ & $\begin{array}{l}358 \\
(48.1)\end{array}$ & $\begin{array}{l}545 \\
(72.6)\end{array}$ & $<0.001$ \\
\hline 20 & $\begin{array}{l}\text { I have had my dentist tell me that I } \\
\text { brush very well }\end{array}$ & Agree & $\begin{array}{l}403 \\
(26.9)\end{array}$ & $\begin{array}{l}127 \\
(17.0)\end{array}$ & $\begin{array}{l}276 \\
(36.8)\end{array}$ & $<0.001$ \\
\hline
\end{tabular}

In Table 3, public and private school mothers held different views on oral health and good oral hygiene. When it comes to mother's awareness about oral health and oral hygiene habits, mothers of children in private schools had better results compared to mothers of children in public schools. However, when questioned about the optimum quantity of toothpaste to be used and about their position when brushing their children's teeth, the replies of mother in public schools were superior to mothers in private schools. 
Table 3

The comparison between public and private school mothers' answers to the knowledge questionnaire

\begin{tabular}{|c|c|c|c|c|c|c|}
\hline \multicolumn{2}{|c|}{ Items } & $\begin{array}{l}\text { The correct } \\
\text { answer }\end{array}$ & $\begin{array}{l}\text { Total } \\
\mathrm{n}=1496\end{array}$ & $\begin{array}{l}\text { Public } \\
\mathrm{n}=745\end{array}$ & $\begin{array}{l}\text { Private } \\
\mathrm{n}=751\end{array}$ & $\begin{array}{l}\mathrm{P}- \\
\text { value }\end{array}$ \\
\hline 1 & $\begin{array}{l}\text { How often should you brush your } \\
\text { child teeth }\end{array}$ & Twice daily & $\begin{array}{l}784 \\
(52.4)\end{array}$ & $\begin{array}{l}350 \\
(47.0)\end{array}$ & $\begin{array}{l}434 \\
(57.8)\end{array}$ & $<0.001$ \\
\hline 2 & Size of brush best for your child & Small & $\begin{array}{l}828 \\
(55.3)\end{array}$ & $\begin{array}{l}398 \\
(53.4)\end{array}$ & $\begin{array}{l}430 \\
(57.3)\end{array}$ & 0.136 \\
\hline 3 & Quantity of paste to be used & pea size & $\begin{array}{l}587 \\
(39.2)\end{array}$ & $\begin{array}{l}362 \\
(48.6)\end{array}$ & $\begin{array}{l}225 \\
(30.0)\end{array}$ & $<0.001$ \\
\hline 4 & $\begin{array}{l}\text { Your position to brush your child's } \\
\text { teeth }\end{array}$ & $\begin{array}{l}\text { By side of the } \\
\text { child }\end{array}$ & $\begin{array}{l}717 \\
(47.9)\end{array}$ & $\begin{array}{l}418 \\
(56.1)\end{array}$ & $\begin{array}{l}299 \\
(39.8)\end{array}$ & $<0.001$ \\
\hline 5 & $\begin{array}{l}\text { Does your child toothpaste have } \\
\text { fluoride }\end{array}$ & Yes & $\begin{array}{l}1099 \\
(73.5)\end{array}$ & $\begin{array}{l}460 \\
(61.7)\end{array}$ & $\begin{array}{l}639 \\
(85.1)\end{array}$ & $<0.001$ \\
\hline 6 & Fluoride content of child paste & $\begin{array}{l}1000 \text { to } 1450 \\
\text { ppm }\end{array}$ & $\begin{array}{l}210 \\
(14.0)\end{array}$ & $\begin{array}{l}59 \\
(7.9)\end{array}$ & $\begin{array}{l}151 \\
(20.1)\end{array}$ & $<0.001$ \\
\hline \multirow[t]{6}{*}{7} & $\begin{array}{l}\text { Four of the following cause tooth } \\
\text { decay. }\end{array}$ & Correct & $\begin{array}{l}1337 \\
(89.4)\end{array}$ & $\begin{array}{l}651 \\
(87.4)\end{array}$ & $\begin{array}{l}686 \\
(91.3)\end{array}$ & 0.013 \\
\hline & o Chocolate (C) & & & & & \\
\hline & o Cheese & & & & & \\
\hline & o Biscuits (C) & & & & & \\
\hline & o Sweets (C) & & & & & \\
\hline & o Soft drink (C) & & & & & \\
\hline 8 & Best time to give sugary snacks & Mealtime & $\begin{array}{l}185 \\
(12.4)\end{array}$ & $\begin{array}{l}64 \\
(8.6)\end{array}$ & $\begin{array}{l}121 \\
(16.1)\end{array}$ & $<0.001$ \\
\hline 9 & $\begin{array}{l}\text { Has child used sweetened baby } \\
\text { bottle or honey dipped pacifier }\end{array}$ & No & $\begin{array}{l}1149 \\
(76.8)\end{array}$ & $\begin{array}{l}540 \\
(72.5)\end{array}$ & $\begin{array}{l}609 \\
(81.1)\end{array}$ & $<0.001$ \\
\hline 10 & Importance of decay in baby teeth & Very important & $\begin{array}{l}1405 \\
(93.9)\end{array}$ & $\begin{array}{l}667 \\
(89.5)\end{array}$ & $\begin{array}{l}738 \\
(98.3)\end{array}$ & $<0.001$ \\
\hline 11 & child's first dental visit & $\begin{array}{l}\text { On getting first } \\
\text { baby tooth }\end{array}$ & $\begin{array}{l}130 \\
(1496)\end{array}$ & $\begin{array}{l}50 \\
(6.7)\end{array}$ & $\begin{array}{l}80 \\
(10.7)\end{array}$ & 0.007 \\
\hline 12 & $\begin{array}{l}\text { If baby teeth decayed, what } \\
\text { treatment would you prefer }\end{array}$ & Fill it & $\begin{array}{l}1227 \\
(82.0)\end{array}$ & $\begin{array}{l}545 \\
(73.2)\end{array}$ & $\begin{array}{l}682 \\
(90.8)\end{array}$ & $<0.001$ \\
\hline
\end{tabular}

Table 4 shows disparities in the replies between public and private school mothers on the mother's attitude questionnaire. Of the 13 assertions, replies to 4 statements $(3,5,11$, and 12) out of the 13 statements showed no statistically significant difference between mothers in public and in private 
schools. In their replies to the 9 reminiscent statements $(1,2,4,6,7,8,9,10$, and 13), the attitude of mothers at private schools was significantly higher than that of mothers at public school.

Table 4

Comparison between public and private schools of the answers from the mother's attitude questionnaire

\begin{tabular}{|c|c|c|c|c|c|c|}
\hline \multicolumn{2}{|c|}{ Items } & \multirow{2}{*}{$\begin{array}{l}\begin{array}{l}\text { The } \\
\text { correct } \\
\text { answer }\end{array} \\
\text { Agree }\end{array}$} & \multirow{2}{*}{$\begin{array}{l}\text { Total } \\
\mathbf{n}=1496 \\
\begin{array}{l}1482 \\
(99.1)\end{array}\end{array}$} & \multirow{2}{*}{$\begin{array}{l}\text { Public } \\
\mathrm{n}=745 \\
732 \\
(98.3)\end{array}$} & \multirow{2}{*}{$\begin{array}{l}\text { Private } \\
\mathbf{n}=751 \\
750 \\
(99.9)\end{array}$} & \multirow{2}{*}{$\begin{array}{l}\begin{array}{l}\text { P- } \\
\text { value }\end{array} \\
0.001\end{array}$} \\
\hline 1 & $\begin{array}{l}\text { We feel it is important that we check our } \\
\text { child's teeth for decay }\end{array}$ & & & & & \\
\hline 2 & $\begin{array}{l}\text { I don't know how to brush my child's teeth } \\
\text { properly }\end{array}$ & Disagree & $\begin{array}{l}857 \\
(57.3)\end{array}$ & $\begin{array}{l}358 \\
(48.1)\end{array}$ & $\begin{array}{l}499 \\
(66.4)\end{array}$ & $<0.001$ \\
\hline 3 & $\begin{array}{l}\text { We feel it is important to check if our child } \\
\text { has brushed his/her teeth }\end{array}$ & Agree & $\begin{array}{l}144 \\
(96.5)\end{array}$ & $\begin{array}{l}717 \\
(96.2)\end{array}$ & $\begin{array}{l}727 \\
(96.8)\end{array}$ & 0.552 \\
\hline 4 & $\begin{array}{l}\text { we don't have time to help brush our } \\
\text { child's teeth daily }\end{array}$ & Disagree & $\begin{array}{l}1009 \\
(67.4)\end{array}$ & $\begin{array}{l}247 \\
(63.4)\end{array}$ & $\begin{array}{l}537 \\
(71.5)\end{array}$ & 0.001 \\
\hline 5 & $\begin{array}{l}\text { It is the responsibility of the dentist to } \\
\text { prevent our child getting tooth decay }\end{array}$ & Disagree & $\begin{array}{l}472 \\
(31.6)\end{array}$ & $\begin{array}{l}220 \\
(29.5)\end{array}$ & $\begin{array}{l}252 \\
(33.6)\end{array}$ & 0.094 \\
\hline 6 & $\begin{array}{l}\text { If our child gets tooth decay, it is by } \\
\text { chance }\end{array}$ & Disagree & $\begin{array}{l}996 \\
(66.6)\end{array}$ & $\begin{array}{l}412 \\
(55.3)\end{array}$ & $\begin{array}{l}584 \\
(77.8)\end{array}$ & $<0.001$ \\
\hline 7 & $\begin{array}{l}\text { It would not make any difference to our } \\
\text { child getting tooth decay, if we helped } \\
\text { him/her brush every day }\end{array}$ & Disagree & $\begin{array}{l}434 \\
(29.0)\end{array}$ & $\begin{array}{l}169 \\
(22.7)\end{array}$ & $\begin{array}{l}265 \\
(35.3)\end{array}$ & $<0.001$ \\
\hline 8 & $\begin{array}{l}\text { It is worthwhile to give our child } \\
\text { sweets/biscuits to behave well }\end{array}$ & Disagree & $\begin{array}{l}775 \\
(51.8)\end{array}$ & $\begin{array}{l}312 \\
(41.9)\end{array}$ & $\begin{array}{l}463 \\
(61.7)\end{array}$ & $<0.001$ \\
\hline 9 & $\begin{array}{l}\text { Tooth decay is a serious problem in baby } \\
\text { teeth }\end{array}$ & Agree & $\begin{array}{l}946 \\
(63.2)\end{array}$ & $\begin{array}{l}406 \\
(54.5)\end{array}$ & $\begin{array}{l}540 \\
(71.9)\end{array}$ & $<0.001$ \\
\hline 10 & $\begin{array}{l}\text { As parents, it is our responsibility to } \\
\text { prevent our child getting tooth decay }\end{array}$ & Agree & $\begin{array}{l}1348 \\
(90.1)\end{array}$ & $\begin{array}{l}648 \\
(87.0)\end{array}$ & $\begin{array}{l}700 \\
(93.2)\end{array}$ & $<0.001$ \\
\hline 11 & $\begin{array}{l}\text { We can prevent tooth decay in our child } \\
\text { by reducing sugary foods and drinks } \\
\text { between meals }\end{array}$ & Agree & $\begin{array}{l}1464 \\
(97.9)\end{array}$ & $\begin{array}{l}732 \\
(98.3)\end{array}$ & $\begin{array}{l}732 \\
(97.6)\end{array}$ & 0.374 \\
\hline 12 & $\begin{array}{l}\text { If we brush our child's teeth daily, we can } \\
\text { prevent our child getting tooth decay in } \\
\text { the future }\end{array}$ & Agree & $\begin{array}{l}1107 \\
(74.0)\end{array}$ & $\begin{array}{l}537 \\
(72.1)\end{array}$ & $\begin{array}{l}570 \\
(75.9)\end{array}$ & 0.092 \\
\hline 13 & $\begin{array}{l}\text { If our child uses a fluoride toothpaste, it } \\
\text { will prevent tooth decay }\end{array}$ & Agree & $\begin{array}{l}1324 \\
(88.5)\end{array}$ & $\begin{array}{l}623 \\
(83.6)\end{array}$ & $\begin{array}{l}701 \\
(93.5)\end{array}$ & $<0.001$ \\
\hline
\end{tabular}

The results in Table 5 demonstrate the associations between demographics of children and mothers and the questionnaires and dmft+DMFT. An effect was established between mothers in public schools and 
lower mean scores on the three questionnaires, but the results were different for their children, who had higher dmft+DMFT and DI scores. Slight differences were seen in questionnaire scores and dmft+DMFT and DI scores when compared by children age and gender. However, as the age of the mothers increased, the questionnaire scores declined while mean $\mathrm{dmft+DMFT}$ and DI increased. Children whose mothers with at least diploma/university degree had greater questionnaire scores and lower dmft+DMFT and DI while the children of mothers with lesser levels of education had higher mean dmft+DMFT and DI. As the income level improved, the questionnaire scores rose while the mean dmft+DMFT and DI descended. 
Table 5

Associations between demographic factors and the scores on the HUDBI questionnaire

\begin{tabular}{|c|c|c|c|c|c|c|}
\hline Variable & Category & HU-DBI & $\begin{array}{l}\text { Mother } \\
\text { Knowledge }\end{array}$ & $\begin{array}{l}\text { Mother } \\
\text { attitude }\end{array}$ & $\mathrm{dmft+DMFT}$ & DI \\
\hline \multirow[t]{3}{*}{ School type } & Public & $\begin{array}{l}10.39 \pm \\
2.75\end{array}$ & $6.13 \pm 1.73$ & $8.51 \pm 1.98$ & $6.70 \pm 4.08$ & $\begin{array}{l}1.36 \pm \\
0.96\end{array}$ \\
\hline & Private & $\begin{array}{l}12.71 \pm \\
2.56\end{array}$ & $6.78 \pm 1.45$ & $9.75 \pm 1.58$ & $4.61 \pm 3.76$ & $\begin{array}{l}0.98 \pm \\
0.88\end{array}$ \\
\hline & $\mathrm{p}$-value & $<0.001$ & $<0.001$ & $<0.001$ & $<0.001$ & $<0.001$ \\
\hline \multirow[t]{3}{*}{ Child age } & $\begin{array}{l}6-9 \\
\text { years }\end{array}$ & $\begin{array}{l}11.41 \pm \\
2.94\end{array}$ & $6.56 \pm 1.67$ & $9.03 \pm 1.94$ & $6.18 \pm 4.44$ & $\begin{array}{l}1.18 \pm \\
0.96\end{array}$ \\
\hline & $\begin{array}{l}10-12 \\
\text { years }\end{array}$ & $\begin{array}{l}11.72 \pm \\
2.85\end{array}$ & $6.33 \pm 1.57$ & $9.25 \pm 1.84$ & $5.04 \pm 3.46$ & $\begin{array}{l}1.15 \pm \\
0.93\end{array}$ \\
\hline & $\mathrm{p}$-value & 0.038 & 0.009 & 0.024 & $<0.001$ & 0.453 \\
\hline \multirow[t]{3}{*}{ Child gender } & Male & $\begin{array}{l}11.11 \pm \\
2.85\end{array}$ & $6.37 \pm 1.73$ & $8.87 \pm 2.01$ & $5.81 \pm 3.97$ & $\begin{array}{l}1.43 \pm \\
0.95\end{array}$ \\
\hline & Female & $\begin{array}{l}11.95 \pm \\
2.89\end{array}$ & $6.53 \pm 1.52$ & $9.36 \pm 1.76$ & $5.51 \pm 4.13$ & $\begin{array}{l}0.93 \pm \\
0.87\end{array}$ \\
\hline & $\mathrm{p}$-value & $<0.001$ & 0.049 & $<0.001$ & 0.158 & $<0.001$ \\
\hline \multirow[t]{3}{*}{ Mother's age } & 40 or less & $\begin{array}{l}12.38 \pm \\
2.74\end{array}$ & $6.88 \pm 1.51$ & $9.59 \pm 1.72$ & $4.80 \pm 3.85$ & $\begin{array}{l}0.97 \pm \\
0.89\end{array}$ \\
\hline & $\begin{array}{l}41 \text { or } \\
\text { more }\end{array}$ & $\begin{array}{l}10.50 \\
\pm 2.75\end{array}$ & $5.91 \pm 1.61$ & $8.54 \pm 1.95$ & $6.75 \pm 4.05$ & $\begin{array}{l}1.42 \pm \\
0.95\end{array}$ \\
\hline & $\mathrm{p}$-value & $<0.001$ & $<0.001$ & $<0.001$ & $<0.001$ & $<0.001$ \\
\hline \multirow[t]{4}{*}{$\begin{array}{l}\text { Mother's } \\
\text { education }\end{array}$} & $\begin{array}{l}\text { High } \\
\text { school }\end{array}$ & $\begin{array}{l}9.66 \pm \\
2.34\end{array}$ & $5.74 \pm 1.63$ & $8.14 \pm 1.83$ & $7.77 \pm 3.69$ & $\begin{array}{l}1.66 \pm \\
0.92\end{array}$ \\
\hline & or less & & & & & \\
\hline & $\begin{array}{l}\text { Diploma/ } \\
\text { University } \\
\text { or higher }\end{array}$ & $\begin{array}{l}13.07 \pm \\
2.36\end{array}$ & $7.03 \pm 1.38$ & $9.92 \pm 1.54$ & $3.96 \pm 3.51$ & $\begin{array}{l}0.77 \pm \\
0.76\end{array}$ \\
\hline & $\mathrm{p}$-value & $<0.001$ & $<0.001$ & $<0.001$ & $<0.001$ & $<0.001$ \\
\hline \multirow[t]{2}{*}{$\begin{array}{l}\text { Monthly } \\
\text { Income }\end{array}$} & Low & $\begin{array}{l}8.77 \pm \\
2.33 \mathrm{a}\end{array}$ & $5.37 \pm 1.72 \mathrm{a}$ & $\begin{array}{l}7.62 \pm 1.99 \\
a\end{array}$ & $\begin{array}{l}8.76 \pm 3.55 \\
a\end{array}$ & $\begin{array}{l}1.83 \pm \\
0.90 \mathrm{a}\end{array}$ \\
\hline & Medium & $\begin{array}{l}11.36 \pm \\
2.07 \mathrm{~b}\end{array}$ & $6.54 \pm 1.46 b$ & $\begin{array}{l}9.05 \pm 1.50 \\
b\end{array}$ & $\begin{array}{l}5.76 \pm 3.68 \\
b\end{array}$ & $\begin{array}{l}1.17 \pm \\
0.86 b\end{array}$ \\
\hline
\end{tabular}




\begin{tabular}{|c|c|c|c|c|c|c|}
\hline Variable & Category & HU-DBI & $\begin{array}{l}\text { Mother } \\
\text { Knowledge }\end{array}$ & $\begin{array}{l}\text { Mother } \\
\text { attitude }\end{array}$ & dmft+DMFT & DI \\
\hline & High & $\begin{array}{l}13.87 \pm \\
2.39 \mathrm{c}\end{array}$ & $7.12 \pm 1.38 \mathrm{c}$ & $\begin{array}{l}10.35 \pm \\
1.49 \mathrm{c}\end{array}$ & $\begin{array}{l}3.23 \pm 3.28 \\
c\end{array}$ & $\begin{array}{l}0.68 \pm \\
0.78 \mathrm{c}\end{array}$ \\
\hline & $p$-value & $<0.001$ & $<0.001$ & $<0.001$ & $<0.001$ & $<0.001$ \\
\hline
\end{tabular}

A multiple linear regression model (adjusted) was used to quantify the impact of school type, mother's age, mother's education, the questionnaire scores, and Debris Index score on the $\mathrm{dmft+DMFT}$ index (Table 6). Overall, $48.6 \%$ of the data variability was accounted for by the model. The $\mathrm{dmft+DMFT}$ scores of children whose mothers had lower educational levels were higher by 0.71 ( $95 \% \mathrm{Cl} 0.31-1.11)$ than those of children whose mothers had higher educational levels. It was predicted that children's dmft+DMFT decreased by $-0.33,-0.16$, and -0.17 for every increase in the questionnaire scores by one. When the Debris Index went up by one, the dmft+DMFT index climbed by 1.43 on average $(95 \% \mathrm{Cl} 1.22-1.64, \mathrm{p}<0.001)$.

Table 6

Model of the impacts of different variables (multiple linear regression, R2=0.495)

\begin{tabular}{|c|c|c|c|c|c|}
\hline \multicolumn{2}{|c|}{ Independent variables } & \multirow{3}{*}{$\begin{array}{l}\beta \pm S E \\
\\
0.013 \pm \\
0.17\end{array}$} & \multicolumn{2}{|l|}{$95 \% \mathrm{Cl}$} & \multirow{2}{*}{$\begin{array}{l}\mathrm{p}- \\
\text { value }\end{array}$} \\
\hline & & & $\begin{array}{l}\text { Lower } \\
\text { bound }\end{array}$ & $\begin{array}{l}\text { Upper } \\
\text { bound }\end{array}$ & \\
\hline \multirow[t]{2}{*}{ School type } & Public & & -0.33 & 0.35 & 0.940 \\
\hline & Private & \multicolumn{3}{|l|}{ Reference } & \\
\hline \multirow[t]{2}{*}{ Mother's age } & 40 or less & $\begin{array}{l}-0.04 \pm \\
0.17\end{array}$ & -0.237 & 0.28 & 0.793 \\
\hline & 41 or more & \multicolumn{3}{|l|}{ Reference } & \\
\hline \multirow{2}{*}{$\begin{array}{l}\text { Mother's } \\
\text { education }\end{array}$} & High school or less & $0.71 \pm 0.20$ & 0.31 & 1.11 & $<0.001$ \\
\hline & $\begin{array}{l}\text { Diploma/University or } \\
\text { higher }\end{array}$ & \multicolumn{3}{|l|}{ Reference } & \\
\hline \multicolumn{2}{|l|}{ HUDBI } & $\begin{array}{l}-0.33 \pm \\
0.05\end{array}$ & -0.42 & -0.24 & $<0.001$ \\
\hline \multicolumn{2}{|c|}{ Mother knowledge } & $\begin{array}{l}-0.16 \pm \\
0.06\end{array}$ & -0.27 & -0.06 & 0.003 \\
\hline \multicolumn{2}{|c|}{ Mother attitude } & $\begin{array}{l}-0.18 \pm \\
0.06\end{array}$ & -0.30 & -0.06 & 0.003 \\
\hline \multicolumn{2}{|l|}{ Debris index } & $1.43 \pm 0.11$ & 1.22 & 1.64 & $<0.001$ \\
\hline
\end{tabular}




\section{Discussion}

Parents' oral health knowledge, attitude, and behavior have an impact on their children's dental health (36), especially the mother, who mostly is the person who attend to her children's oral hygiene needs. As a result, the purpose of this study was to determine the level of oral health-related knowledge, attitudes, and behavior among a group of mothers with children aged 6 to 12 years, as well as to investigate the relationship between mothers' socioeconomic status and their knowledge, attitudes, and behavior regarding their own and their children's dental health. The impact of mothers' oral health knowledge and attitudes on their children's dental caries status (dmft/DMFT) and oral hygiene condition (DI) were also investigated in this study.

In comparison to mothers of public primary school children, mothers whose children attend private primary schools have higher questionnaire scores on average (HU-DBI, knowledge, and attitude). The study's findings can be explained by the fact that most private schools provide oral health education to children and their parents, but most public schools do not. These arguments are supported by a 2017 study conducted in Riyadh, which indicated that oral health educational sessions in private schools improved schoolchildren's oral health awareness (37). Schools are accountable for more than simply providing education; they are also responsible for informing parents and students about other aspects such as oral health. According to the Haloi et al study, the type of school has a substantial impact on oral health promotion, with schoolteachers in private schools having relatively high oral health knowledge and attitudes (38). In contrast to our findings, an Indian study found that the majority of moms of children in private schools had a mediocre understanding of oral health (38). In addition, an Iranian study indicated that mothers of children in public schools had a good understanding and attitude toward dental health (39). However, neither study revealed the participants' socioeconomic background, which could have a substantial confounding effect on the relationship between school type (private vs. public) and oral health-related knowledge, attitudes, and behavior.

Children in private primary schools have lower $\mathrm{dmft} / \mathrm{DMFT}$ and DI mean scores on average than children in public primary schools, according to our research. These findings are similar to those of a 2016 study in Nigeria, which found that children in private schools scored lower on the DMFT than children in public schools (40). In India, it was discovered in 2016 that children in private schools (12-13 years old) had a lower DMFT mean score than kids in public schools (41). In Brazil in 2004, children in public schools had higher dmft/DMFT than children in private schools, with a statistically significant difference (42). On the contrast, there was no statistically significant difference in $\mathrm{dmft} / \mathrm{DMFT}$ scores between public and private school students in Port Harcourt, Nigeria, despite private school students having lower dmft/DMFT scores (43).

In comparison to moms with lesser education and/or income, mothers with higher education and/or income had better oral health related knowledge, attitudes and behavior, and their children had fewer cavities and lower DI. These results could be explained by the fact that women have a significant role in their children's dental health, and that their high levels of education enable them to seek out the best oral 
hygiene practices. Mothers with a higher level of education are likely to be more aware of the risks of neglecting oral health and the oral disorders that may harm their children. In terms of oral health, educated mothers can use the internet, social media, and electronic periodicals to find reliable oral health information. Higher-educated mothers recognize the necessity of teaching their children how to brush their teeth and to examine them thereafter. This may motivate children to brush their teeth thoroughly and maintain good oral hygiene. Another possibility is that children of mothers with higher levels of education and income have more frequent dental checkups. Most highly educated mothers took their infants to the dentist when they were six to twelve months old, whereas the majority of illiterate parents took their children to the dentist only when they were in pain (44). According to other studies (45), children's oral health is directly related to their monthly family income, and children of low-income mothers use fewer dental services than children of high-income mothers. Our findings are in line with two Saudi Arabian studies that found a link between educational and financial levels, as well as oral hygiene knowledge and attitudes $(18,46)$. A higher prevalence of caries was connected to a lower parental educational level and a lower family income (47). As a result, it's vital to inform low-income mothers about their children's dental care options.

\section{Conclusion}

The study results revealed that the level of oral health- related knowledge, attitudes, and behavior of the mothers toward their own and their children (6-12 years), with high educational level a were higher and consequently their children had better oral health status. Overall, participated mothers need to increase the level of oral health knowledge and improve their oral health attitudes and behavior. Therefore, there is a need for increase comprehensive oral health education and oral health promotion programs among mothers in Jeddah, Saudi Arabia. Moreover, implementation of awareness campaign of oral health education programs targeted at pre-school and primary school children to encourage them for good oral health attitude and behaviors, through schools' extra-curricular activities and electronic social media. Additional studies are required to evaluate mothers' awareness regarding oral health in different cities in Saudi Arabia which would help in planning and designing public health campaign.

\section{Abbreviations}

HU-DBI- Hiroshima University- Dental Behavioral Inventory questionnaire

CVI - The Content Validity Index

dmft - Decayed, Missed, Filled teeth for the primary dentition

DMFT - Decayed, Missed, Filled teeth for the permanent dentition

DI - Debris Index component

OHI-S - Simplified Oral Hygiene Index 
ICC - Intra-class Correlation

SPSS - Statistical Package for Social Science

Cl - Confidence Intervals

\section{Declarations}

\section{Ethical approval}

The research protocol was approved by Research Ethics Committee at the Faculty of Dentistry, King Abdulaziz University, Jeddah, Saudi Arabia with proposal number (008-16). In addition, approval was obtained from the local School Health and Education Directorate Authority; Ministry of Education; to provide the primary schools' lists in Jeddah city districts and to implement the research among the schools. A written consent form was obtained from all participant before participating in the research.

\section{Consent for Publication}

Not applicable

\section{Data availability}

The datasets used and/or analyzed during the current study are available from the corresponding author on reasonable request.

\section{Competing Interests}

The authors did not have any conflict of interest related to this study.

\section{Funding}

This project was funded by the Deanship of Scientific Research (DSR), King Abdulaziz University, Jeddah, Kingdom of Saudi Arabia under grant number G: 419/165/1440.

\section{Author's Contributions}

We declare that this work was done by the author(s) named in this article. The study's conception and design were done by NA and EE; acquisition of data by DQ; analysis and/or interpretation of data by OF. Drafting of the manuscript was by DQ and RA while revising the manuscript was by NA, EE and RA. All authors of the manuscript have read and agreed to its content and are accountable for all aspects of the accuracy and integrity of the manuscript.

\section{Acknowledgements}


The authors acknowledge with thanks DSR for technical and financial support.

This research published from the thesis of Dr. Duha Qahtan.

\section{References}

1. Al Agili DE. A systematic review of population-based dental caries studies among children in Saudi Arabia. The Saudi dental journal. 2013;25(1):3-11.

2. Mathur A, Gupta T. Oral health attitude knowledge behavior and consent towards dental treatment among school children. Journal of orofacial research. 2011:06-10.

3. Flinck A, Källestål C, Holm AK, Allebeck P, Wall S. Distribution of caries in 12-year-old children in Sweden. Social and oral health-related behavioural patterns. Community dental health. 1999;16(3):160-5.

4. Petersen PE. Sociobehavioural risk factors in dental caries - international perspectives. Community dentistry and oral epidemiology. 2005;33(4):274-9.

5. Källestål C, Wall S. Socio-economic effect on caries. Incidence data among Swedish 12-14-year-olds. Community dentistry and oral epidemiology. 2002;30(2):108-14.

6. Tinanoff N. Dental caries risk assessment and prevention. Dental clinics of North America. 1995;39(4):709-19.

7. Elley KM, Langford JW. The use of a classification of residential neighbourhoods (ACORN) to demonstrate differences in dental health of children resident within the south Birmingham health district and of different socio-economic backgrounds. Community dental health. 1993;10(2):131-8.

8. Kuppuswamy VL, Murthy S, Sharma S, Surapaneni KM, Grover A, Joshi A. Oral hygiene status, knowledge, perceptions and practices among school settings in rural South India. Oral health and dental management. 2014;13(1):146-54.

9. Quadri FA, Hendriyani H, Pramono A, Jafer M. Knowledge, attitudes and practices of sweet food and beverage consumption and its association with dental caries among schoolchildren in Jazan, Saudi Arabia. Eastern Mediterranean health journal $=$ La revue de sante de la Mediterranee orientale $=$ al Majallah al-sihhiyah li-sharq al-mutawassit. 2015;21(6):403-11.

10. Al-Meedani LA, Al-Dlaigan YH. Prevalence of dental caries and associated social risk factors among preschool children in Riyadh, Saudi Arabia. Pakistan journal of medical sciences. 2016;32(2):452-6.

11. Gurunathan D, Moses J, Arunachalam SK. Knowledge, Attitude, and Practice of Mothers regarding Oral Hygiene of Primary School children in Chennai, Tamil Nadu, India. International journal of clinical pediatric dentistry. 2018;11(4):338-43.

12. Mattila ML, Rautava P, Sillanpää M, Paunio P. Caries in five-year-old children and associations with family-related factors. Journal of dental research. 2000;79(3):875-81.

13. Elena B, Petr L. Oral health and children attitudes among mothers and school teachers in Belarus. Stomatologija Baltic Dental and Maxillofacial Journal. 2004;6:40-3. 
14. Castilho AR, Mialhe FL, Barbosa Tde S, Puppin-Rontani RM. Influence of family environment on children's oral health: a systematic review. Jornal de pediatria. 2013;89(2):116-23.

15. Alhabdan YA, Albeshr AG, Yenugadhati N, Jradi H. Prevalence of dental caries and associated factors among primary school children: a population-based cross-sectional study in Riyadh, Saudi Arabia. Environmental health and preventive medicine. 2018;23(1):60.

16. Klein $\mathrm{H}$. The family and dental disease; dental disease (DMF) experience in parents and offspring. Journal of the American Dental Association (1939). 1946;33:735-43.

17. Ringelberg ML, Matonski GM, Kimball AW. Dental caries-experience in three generations of families. Journal of public health dentistry. 1974;34(3):174-80.

18. Hamasha AA, Rasheed SJ, Aldosari MM, Rajion Z. Parents knowledge and awareness of their children's oral health in Riyadh, Saudi Arabia. The Open Dentistry Journal. 2019;13(1).

19. Carlson DA BG VA, Brasili DS., inventorCavium Network LLC, assignee. Random number genertor. United Sates patent US 6,954,770 2005.

20. Kawamura M. [Dental behavioral science. The relationship between perceptions of oral health and oral status in adults]. Hiroshima Daigaku shigaku zasshi The Journal of Hiroshima University Dental Society. 1988;20(2):273-86.

21. Mekhemar M, Conrad J, Attia S, Dörfer C. Oral Health Attitudes among Preclinical and Clinical Dental Students in Germany. International journal of environmental research and public health. 2020;17(12).

22. Almarek F, Assery M, Baseer M. Oral health attitudes and behavior among health professionals in Riyadh City, Saudi Arabia. Journal of International Oral Health. 2017;9(4):156-64.

23. Alam Moheet I, Farooq I. Self-reported differences between oral health attitudes of pre-clinical and clinical students at a dental teaching institute in Saudi Arabia. The Saudi dental journal. 2013;25(4):149-52.

24. Jaramillo JA, Jaramillo F, Kador I, Masuoka D, Tong L, Ahn C, et al. A comparative study of oral health attitudes and behavior using the Hiroshima University-Dental Behavioral Inventory (HU-DBI) between dental and civil engineering students in Colombia. Journal of oral science. 2013;55(1):23-8.

25. Vangipuram S, Rekha R, Radha G, Pallavi S. Assessment of oral health attitudes and behavior among undergraduate dental students using Hiroshima University Dental Behavioral Inventory HUDBI. Journal of Indian association of public health dentistry. 2015;13(1):52.

26. Khalid K, Naidoo S, Elamin F. Oral health behaviours and attitudes using the modified Arabic Version of Hiroshima University-Dental Behavioural Inventory (HU-DBI) among sudanese dental students. Int J Dentistry Oral Sci. 2016;3(9):326-30.

27. Carrasco FL CJ, Serrano CY, et al. Comparison of the HU-DBI index between areas of health sciences and other areas of the University of Cuenca, 2016. J Dent Health Oral Disord Ther. 2018;9(3):239-43.

28. Al-Wesabi AA, Abdelgawad F, Sasahara H, El Motayam K. Oral health knowledge, attitude and behaviour of dental students in a private university. BDJ open. 2019;5:16. 
29. Noorani T NJ, Abdul Ghani NN, Moheet I. Self-reported differences in oral health attitudes and behaviors of health-care students at a University in Malaysia. European Journal of General Dentistry. 2018;7(1).

30. Prabhu A, Rao AP, Reddy V, Ahamed SS, Muhammad S, Thayumanavan S. Parental knowledge of pre-school child oral health. Journal of community health. 2013;38(5):880-4.

31. Lenčová E, Dušková J. Oral health attitudes and caries-preventive behaviour of Czech parents of preschool children. Acta medica academica. 2013;42(2):209-15.

32. WHO. Process of translation and adaptation of instruments: World Health Organization [cited 2009. Available from: http://www. who. int/substance_abuse/research_tools/translation/en/. 2009]2009.

33. Lynn MR. Determination and quantification of content validity. Nursing research. 1986;35(6):382-5.

34. Polit DF, Beck CT, Owen SV. Is the CVI an acceptable indicator of content validity? Appraisal and recommendations. Research in nursing \& health. 2007;30(4):459-67.

35. WHO. World Health Organization Oral Health Surveys Basic Methods. 4th ed. Geneva: WHO; 1997. 40-52 p.

36. Al-Zahrani AM, Al-Mushayt AS, Otaibi MF, Wyne AH. Knowledge and attitude of Saudi mothers towards their preschool children's oral health. Pakistan journal of medical sciences. 2014;30(4):7204.

37. Al Saffan AD, Baseer MA, Alshammary AA, Assery M, Kamel A, Rahman G. Impact of Oral Health Education on Oral Health Knowledge of Private School Children in Riyadh City, Saudi Arabia. Journal of International Society of Preventive \& Community Dentistry. 2017;7(Suppl 3):S186-s93.

38. Haloi R, Ingle NA, N K. Caries Status of Children and Oral Health Behavior, Knowledge and Attitude of Their Mothers and Schoolteachers in Mathura City. J Contemp Dent. 2012;2(3):78-83.

39. Saied-Moallemi Z, Virtanen JI, Ghofranipour F, Murtomaa H. Influence of mothers' oral health knowledge and attitudes on their children's dental health. European archives of paediatric dentistry: official journal of the European Academy of Paediatric Dentistry. 2008;9(2):79-83.

40. SOROYE MO, OB. B. An Oral Health Status of Children in Government and Private Secondary Schools in Lagos State, Nigeria. NJDR. 2016;1(1\&2):34-40.

41. Abraham A, Pullishery F, R R. Dental caries and calculus status in children studying in Government and Private Schools in Malappuram, Kerala, India.. IAIM. 2016;3(3):35-41.

42. Hoffmann RH, Cypriano S, Sousa Mda L, Wada RS. [Dental caries experience in children at public and private schools from a city with fluoridated water]. Cadernos de saude publica. 2004;20(2):5228.

43. Eigbobo J, Alade G. Dental caries experience in primary school pupils in Port Harcourt, Nigeria Sahel Med J. 2017;20(4):179-86.

44. Almulhim B, Alamro B. Knowledge and attitude toward oral health practice among the parents in Riyadh city. J Indian Acad Dent Spec Res. 2016(3):14-6. 
45. Akpabio A, Klausner CP, Inglehart MR. Mothers'/guardians' knowledge about promoting children's oral health. Journal of dental hygiene: JDH. 2008;82(1):12.

46. Salama AA, Konsowa EM, SH A. Mothers' knowledge, attitude, and practice regarding their primary school children's oral hygiene. Menoufia Med J. 2020;32(1):11-7.

47. Cianetti S, Lombardo G, Lupatelli E, Rossi G, Abraha I, Pagano S, et al. Dental caries, parents educational level, family income and dental service attendance among children in Italy. European journal of paediatric dentistry. 2017;18(1):15-8. 